\title{
Multispectral Fluorescence Imaging
}

\author{
Lanlan Zhou and Wafik S. El-Deiry \\ Department of Bioengineering, University of Pennsylvania School of Engineering and Applied Science, Philadelphia, Pennsylvania; and \\ Hematology-Oncology Division, Department of Medicine, Genetics, and Pharmacology, Abramson Comprehensive Cancer Center, \\ University of Pennsylvania School of Medicine, Philadelphia, Pennsylvania
}

\begin{abstract}
Multispectral fluorescence imaging (MSFI) is a rapidly growing field with broad applications in both preclinical and clinical settings. Application of this novel technology in small-animal imaging and microscopy produces enhanced sensitivity and reliable quantification and resolves multiple simultaneous signals. MSFI flow cytometry can quantify multiple fluorescent parameters with morphologic or subcellular spatial details on millions of cells. MSFI has the potential to improve the accuracy of disease detection or differentiation and intrasurgical metastatic diagnosis, guide neurosurgeries, and monitor treatment response.

Key Words: multispectral fluorescence imaging; multiplexing; spectral unmixing; small-animal imaging; microscopy; flow cytometry
\end{abstract}

J Nucl Med 2009; 50:1563-1566

DOI: 10.2967/jnumed.109.063925

Multispectral imaging (MSI) is the synergistic combination of imaging and spectroscopy. Color is the appearance of a light most affected by wavelength $(\lambda)$ and the observers' visual system. Objects with similar colors are not necessarily the same. The simplest example is the spectrally pure yellow and the mixture of red and green, which have completely different spectral content but precisely the same redgreen-blue (RGB) coordinates (1). Spectroscopy is the technique of breaking light down into its composite colors to identify the composition of the object. Distinguished from conventional RGB full-color imaging, MSI can obtain a high-resolution optical spectrum for each image pixel, resulting in a series of images of the same field of view that are acquired at different wavelengths. These can be stacked in 3-dimensional datasets or a cube (x, y, and $\lambda$ ). After proper calibration, quantitative images of individual analytes can be generated.

Excellent reviews of MSI instrumentation and data analysis exist $(1,2)$. Briefly, a camera is used to acquire the spatial information, and the spectral information is gained by scanning a dispersive element to record spectra for each image. Electronic tunable filters such as an acousto-optic tunable filter and liquid crystal tunable filters (LCTFs) are preferable to mechanically scanning dispersive devices (filter wheels, monochromators) because they are quiet, fast, compact, and stable and demonstrate increased spectral selectivity, spectral purity, and flexibility. The disadvantages of electronic tunable filters include low light

Received Jun. 18, 2009; revision accepted Aug. 19, 2009.

For correspondence or reprints contact: Wafik S. El-Deiry, 415 Curie Blvd., 437A, Clinical Research Building, Philadelphia, PA 19104

E-mail: wafik@mail.med.upenn.edu

COPYRIGHT @ 2009 by the Society of Nuclear Medicine, Inc. throughput, photobleaching, and inappropriate capture if significant sample or camera movement occurs during the acquisition or if high temporal resolution is needed to capture certain events (e.g., calcium signaling transients) (3). The typical method for data analysis of MSI is a least-squares-fitting linear unmixing approach with additional constraints (such as nonnegativity), resulting component images, and a final composite image. This approach is limited to spectral analysis only, whereas combining the rich spatial information in the images with the spectral data is a more powerful and evolving field (1). Multispectral fluorescence imaging (MSFI) coupled with microscopy and flow cytometry are commercially available now $(4,5)$.

MSI technologies have been widely used in the fields of astronomy, geology, agriculture, industry, and forensics $(1,2)$. With the vast development of filters, detectors, data-analysis techniques, interdisciplinary approaches, and fluorescent dyes, MSFI is a rapidly growing field with applications in cell biology, preclinical drug development, and clinical pathology. Combining MSFI with small-animal imaging and microscopy produces enhanced sensitivity, reliable quantitation, and resolved multiple simultaneous signals $(3,6)$. MSFI is particularly useful for analyzing objects that have multiple fluorescent labels that may have similar RGB color or that may be localized in the same or spatially overlapping compartments; this modality is also effective for evaluating objects that have strong whole-animal autofluorescence (Fig. 1) (4,7,8). Here we review a selection of preclinical and clinical studies describing the use of MSFI applications.

\section{PRECLINICAL APPLICATION OF MFSI}

\section{To Assess Tumor Vascularization and Monitor Anti- angiogenic Therapeutic Response}

Previous studies imaged tumor angiogenesis with fluorescent proteins $(9,10)$. MSFI provides a powerful, noninvasive means by which to assess tumor vascularity and to monitor the response to antiangiogenic therapy. For example, in mice treated with sunitinib, a near-infrared dye-labeled monoclonal antibody (mAb)-based probe targeting vascular endothelial growth factor receptor-2 was imaged in vivo using an LCTF-based Maestro MSFI system (CRI Inc.). Tumor fluorescence intensity was quantified and normalized after autofluorescence removal. This study demonstrates that MSFI provides rapid, noninvasive, and longitudinal assessment of the efficacy of novel angiogenesis-directed therapies in preclinical models of cancer (11). To overcome constraints associated with exogenously administered vascular imaging probes such as high cost, toxicity, inconsistent delivery, differential bioavailability among animals, and high tumor accumulation resulting from vessel leakiness, a novel method to noninvasively image tumor vascularization in fluorescent tumors without exogenous imaging probes was introduced. LCTF-based Maestro and Nuance 
A

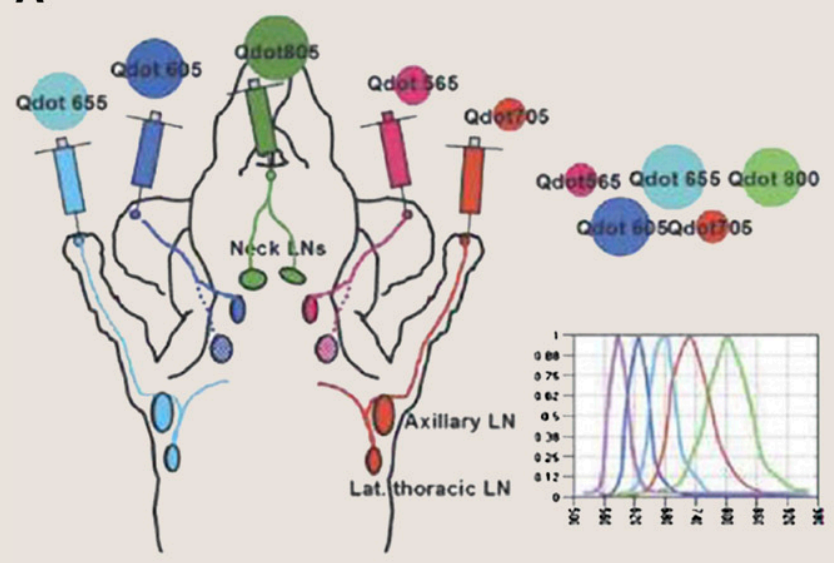

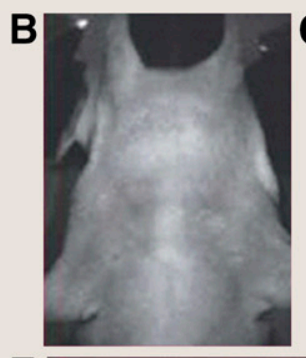
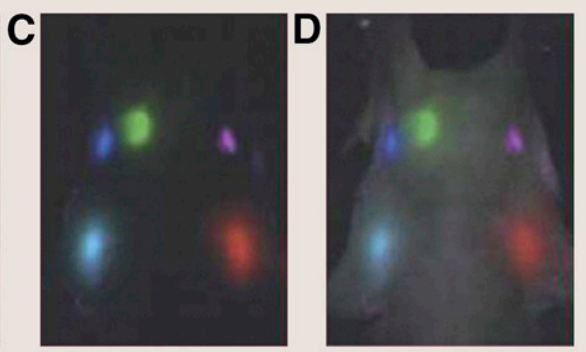

E
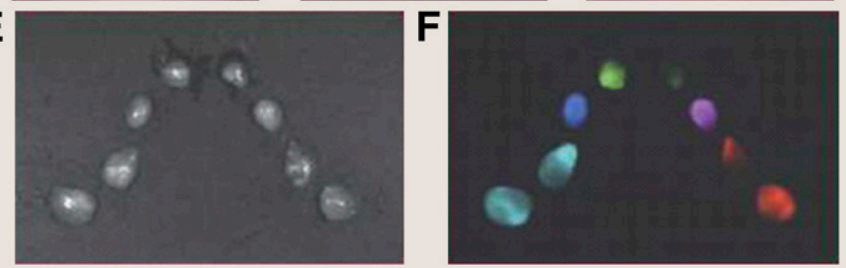

FIGURE 1. Five-color spectrally unmixed quantum dot detection of lymphatic system anatomy. (A) Schematic illustration of 5-color quantum dot lymphatic injection sites and draining destinations of spectral fluorescence imaging. (B) Autofluorescence image of mouse. (C) Composite pseudocolored detection of draining lymph nodes after spectral unmixing. (D) Image merge of $B$ and C. Reflectance $(E)$ and spectrally unmixed and pseudocolored $(F)$ images of surgically dissected lymph nodes arranged in same geometry as in intact mouse. This figure originally appeared in an article by Richard M. Levenson et al. in ILAR Journal 49(1) (8). It is reprinted with permission from the ILAR Journal, Institute for Laboratory Animal Research, The National Academies, Washington DC (www.national-academies.org/ilar).

systems (CRI Inc.) were used for in vivo MSFI to study the biology of tumor angiogenesis and to monitor the effects of antiangiogenic therapies with bevacizumab. In a mouse model bearing tumors expressing red fluorescent protein, the spectral fluorescence signatures of vascular and avascular components allow for the tumor vasculature to be imaged and quantified without contrast agents (Fig. 2). This technique provides real-time imaging of tumor vascularization and monitoring of the antiangiogenic therapeutic response in mice, without the concerns specific to an exogenously administered probe (12).

\section{To Generate Region-Selective Brain Maps of \\ Vascularized Brain Parenchyma}

A fluorescein isothiocyanate-derivatized tomato lectin-based molecular imaging probe was combined with the Maestro system to generate region-selective brain maps of vascularized brain parenchyma ex vivo in rats and rabbits. Images were unmixed to get rid of brain tissue autofluorescence and resolve arterial blood and specific imaging probe fluorescence signal. After further refinement of imaging probes and metrics, this novel preoperative endovascular brain-mapping approach may facilitate neurosurgical guidance and therefore improve clinical outcomes after neurosurgical resections (13).

\section{Automatable Analysis of Multiplexed Immunolabeled Samples}

More than 6 markers can be quickly differentiated from each other and from autofluorescence using MSFI, thereby allowing for the localization of multiple probes and avoiding the need for serial sections. For example, Mansfield et al. performed 6-marker analyses in 1 sample with MSFI. Quantitative and marker-specific image data were presented for integrative system biologic studies. The drawbacks of this approach are that it is too much work to prepare a series of controls of all of the combinations of markers and the method is less optimal for membrane or cytoplasmic marker analyses (4).

\section{To Differentiate Tissue Type and Provide Cellular-Level Diagnostic Information}

The slit-scan confocal microendoscope uses a catheter based on a fiber-optic imaging bundle. This multispectral system uses a prism as a dispersive element to collect a full multispectral image with spectral resolution of $2.9 \mathrm{~nm}$ at $500 \mathrm{~nm}$ and $8.4 \mathrm{~nm}$ at $750 \mathrm{~nm}$. Human ovary and esophagus and mouse peritoneal wall and liver stained with acridine orange and rat intestine tagged with 4-(4-(dihexadecylamino)styryl)- $N$-methylpyridinium iodine (Invitrogen) were imaged ex vivo. This method of MSFI can distinguish tissue types and acquire high-quality images; it is minimally invasive and has the potential to provide cellular-level in vivo diagnostic information as a clinical diagnostic instrument (14).

\section{Combining MSFI with Quantum Dots (MSFI-QDs)}

Quantum dots (QDs) are novel nanocrystal fluorophores with constant broad excitation spectra, sharp and symmetric tunable emission spectra, improved brightness, superior photostability, and simultaneous excitation of multiple fluorescence colors. QDs have been successfully applied in various imaging applications for in vitro diagnostics and in vivo imaging because of these unique optical properties. MSFI-QDs can efficiently remove background and precisely delineate weak spectral signatures to present ultrasensitive and multiplexed imaging of molecular targets in vivo $(15,16)$. Full reviews on MSFI-QDs in multiplexed immunohistochemistry and in situ hybridization (ISH) are available $(17,18)$. Another use of MSFIQDs is as a feasible substitute for microarray analysis. In this instance, multiplexed QD-labeled oligonucleotide probes can be used for ISH in human biopsies. This technique was tested in a study in which single and multiplexed QD-ISH was performed in samples with acute leukemia and follicular lymphoma. Spectral unmixing enables the separation of spatially colocalized signals. MSFI-QDs allow the quantitative characterization of multiple-gene expression, thereby making them useful for the analysis of processed human clinical tissues (19). MSFI-QDs were also tested using streptavidin-conjugated QDs to detect up to 7 signals in tonsil and lymphoid tissue. Slides were analyzed using confocal laser scanning microscopy. Five streptavidin-conjugated QDs were used on the same tissue section and could be analyzed simultaneously on routinely processed sections (Fig. 3). This multiplexing method has the potential to uncover the clinically relevant multidimensional cellular interactions that underlie diseases (20). 

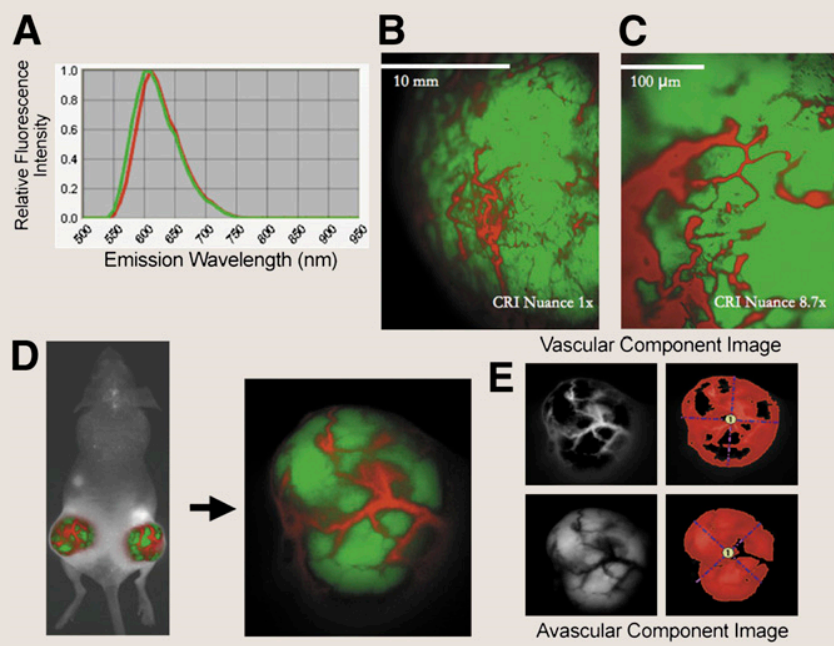

FIGURE 2. Noninvasive imaging of tumor vasculature using multispectral unmixing in fluorescent tumors. (A) Spectral fluorescent signatures of vascular components (emission wavelength maximum or peak emission $\left[\mathrm{Em}_{\text {max }}\right]=$ $620 \mathrm{~nm}$, red spectra) and avascular components $\left(\mathrm{Em}_{\max }=\right.$ $610 \mathrm{~nm}$, green spectra). (B) Image of fluorescent tumor taken using CRI Nuance camera. (C) High-magnification image of same tumor area as B shows ability to detect capillaries (5-10 $\mu \mathrm{m}$ in diameter) within fluorescent tumors using multispectral unmixing. (D) Multispectral unmixing of macroscopic whole-body image of mouse with fluorescent tumors on both rear flanks (left panel). Enlarged image of left rear flank tumor (right panel). (E) Custom threshold region of interest is created in vascular and avascular component images using CRI software. Vascular index is then calculated by dividing avascular component total area $\left(\mathrm{mm}^{2}\right)$ by vascular component total area $\left(\mathrm{mm}^{2}\right)$. Reprinted from (12) (c) 2008 BioTechniques, Used by Permission.

\section{CLINICAL APPLICATION OF MSFI}

\section{Combining MSFI with 2-Photon Excitation (TPE) \\ to Detect Cutaneous Tumors}

TPE fluorescence microscopy offers high spatial resolution, deep penetration, and low photodamage. To detect cutaneous tumors, a combined TPE and MSFI system (MTPE) was used to identify different intrinsically fluorescent molecules (nicotinamide adenine dinucleotide, tryptophan, keratins, melanin, elastin, cholecalciferol, and others). This technique provides functional information correlated with tissue structure in physiologic and pathologic states. MTPE is effective in differentiating healthy skin from basal cell carcinoma (21). As more samples are analyzed, it should be possible to differentiate between different types of malignancies as well.

\section{Application of MSI Flow Cytometry}

MSI flow cytometry can quantify multiple fluorescent parameters with morphologic or subcellular spatial details on millions of cells $(5,22)$. Nuclear translocation of NFKB initiates the transcription of host defense-related critical genes. To quantitatively measure the translocation of signaling molecules from the cytoplasm to the nucleus in cells, cells were stained with fluorescein isothiocyanate-conjugated anti-NFKB and 7-AAD (DNA stain) and imaged by flow cytometry. A similarity score that quantifies the correlation of the spatial distribution of the stained, translocating signaling molecule with nuclear staining was generated for each cell using Pearson correlation coefficients to assess the nuclear translocation. This approach represents an
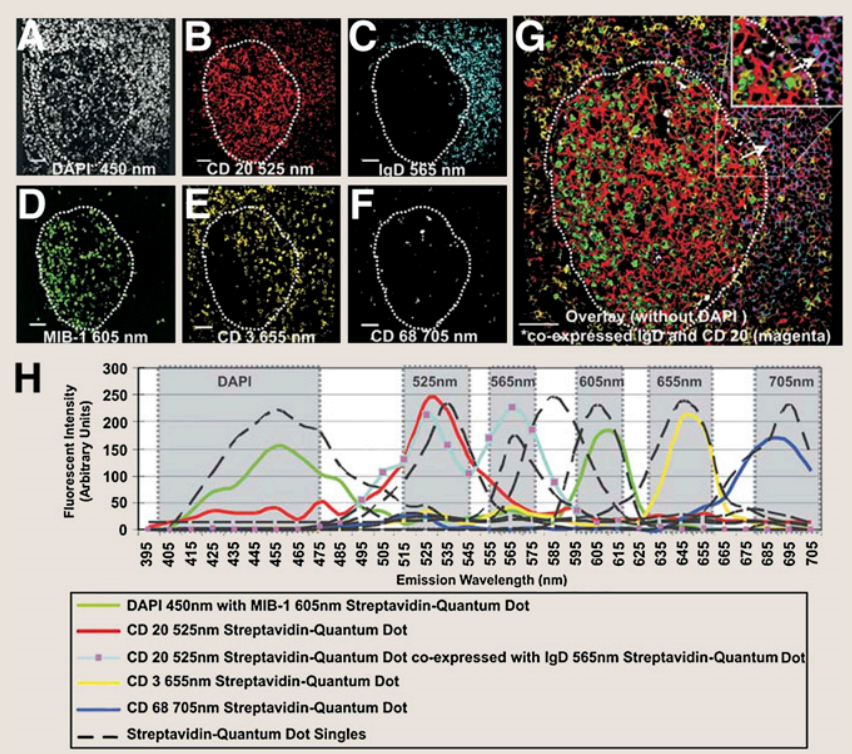

FIGURE 3. Quintuplet streptavidin-conjugated quantum dots multiplex and emission fingerprint of multiplexed quantum dots (germinal center outlined with dotted line). (A) 4,6-diamino-2-phenylindole nuclear counterstain, $450 \mathrm{~nm}$ (white). (B) CD 20 streptavidin-conjugated quantum dot 525 $\mathrm{nm}$ (red). (C) IgD streptavidin-conjugated quantum dot, 565 $\mathrm{nm}$ (cyan). (D) MIB-1 streptavidin-conjugated quantum dot, $605 \mathrm{~nm}$ (green). (E) CD 3 streptavidin-conjugated quantum dot, $655 \mathrm{~nm}$ (yellow). (F) CD 68 streptavidin-conjugated quantum dot, $705 \mathrm{~nm}$ (white). (G) Overlay of B-F including $\operatorname{lgD}$ (cyan) and CD20 (red) coexpression (magenta; arrows and inset). (H) Emission profile generated from $\lambda$-scan from 395 to $715 \mathrm{~nm}$. Dotted lines are single emission profile from 4,6-diamino-2-phenylindole alone or each of streptavidinconjugated quantum dots alone. Solid-colored lines are indicated emission profiles from quintuplet-labeled tonsil section, and gray areas define band pass filters used. Profiles with same color indicate colocalization. Scale bar is $50 \mathrm{~mm}$. Reprinted from (20) by permission from Macmillan Publishers Ltd: Modern Pathology copyright 2006.

advance over conventional microscopy or biochemical fractionation and Western blotting for high throughput single-cell or subpopulation analysis $(23,24)$. MSI flow cytometry can not only distinguish live cells from cells in the early or late phases of apoptosis or from necrotic cells (25) but also accurately quantitate apoptosis based on morphologic characterization and allow a more complete and robust analysis of apoptosis than conventional microscopy (26). Covalent deposition of $\mathrm{C} 3$ fragments $(\mathrm{C} 3 \mathrm{~b} / \mathrm{iC} 3 \mathrm{~b})$ on cells is closely associated with cell-bound chimeric humanized anti-CD20 mAb rituximab (RTX). To quantitatively analyze colocalization of C3 with RTX, Raji cells were stained with fluorescently labeled RTX and mAbs specific for $\mathrm{C} 3 \mathrm{~b} / \mathrm{iC} 3 \mathrm{~b}$ and then imaged in flow and analyzed for colocalization of fluorescent probes. Analysis verified that most deposited C3b/iC $3 b$ is colocalized with bound RTX (27).

\section{Autofluorescence Analysis in MSFI}

MSFI of oral tissue autofluorescence in vivo with a 4-channel multispectral digital microscope can improve the detection of oral neoplasia. Decreased blue-green autofluorescence and increased red autofluorescence in lesions were observed in patients with histologically confirmed neoplasia (28). MSFI of the ocular fundus autofluorescence may differentiate age-related macular degeneration 
and diabetic retinopathy (RD). Autofluorescence in age-related macular degeneration patients was dominated by the red emission, whereas RD green-shifted. Lipofuscin is the dominant fluorophore in the red channel. The green shift in RD may be related to increased advanced glycation end product concentrations (29). MSFI of sentinel lymph node autofluorescence during surgery can provide a rapid method to verify whether a tumor has metastasized to help determine follow-up treatment. For example, to evaluate lymph nodes in colorectal and gastric tumors, autofluorescence microspectroscopy and MSI autofluorescence microscopy have been used to analyze the autofluorescence emission of metastatic lymph node sections. The autofluorescence pattern and the emission spectrum of metastatic lymph nodes can distinguish them from the normal ones (30). MSFI of autofluorescence has the potential for detecting oral neoplasia, discriminating fundus autofluorescence fluorophores and intrasurgical diagnosis of metastatic lymph nodes.

\section{Spatial-Spectral Analysis in MSFI}

Using exclusive spectral information from an LCTF-based MSFI system with image analysis software (Environment for Visualizing Images) can identify malignant urothelial cells in urine cytology specimens, with a sensitivity and specificity of $82 \%$ and $81 \%$, respectively, or a combined sensitivity and specificity of $73 \%$ (31). To increase the accuracy and specificity of the diagnosis and follow-up of bladder cancer, the spatial-spectral features of multispectral image stacks acquired with the same MSFI system were analyzed using the GENetic Imagery Exploitation (GENIE) package (Los Alamos National Laboratory). GENIE showed a combined sensitivity and specificity of $85 \%$ and $95 \%$ in samples from 2 separate institutes. Furthermore, GENIE showed efficiency superior to a cytopathologist with respect to predicting the follow-up results (32). MSFI of fineneedle aspirations of follicular adenoma and parathyroid adenoma with the Nuance system and spatial-spectral analysis with CRI-MLS software can differentiate and classify morphologically similar lesions. The best spatial-spectral imaging solution has a sensitivity of $98.5 \%$, a specificity of $96.1 \%$, and a positive predictive value of $98.6 \%$ (33). Spatial-spectral analysis is a promising tool for improving the detection of bladder carcinoma when morphology is not enough for a definitive diagnosis and differentiation of lesions with subtle cytomorphologic differences.

We have reviewed how MSFI can increase sensitivity and reliable quantification in preclinical and clinical applications. MSFI has been widely used in applications ranging from flow cytometry, microscopy, endoscopy, and small-animal imaging to human imaging. With the development of imaging devices, data analysis technologies, and fluorescent dyes, MSFI has the potential to improve the accuracy of disease diagnosis and predict treatment response.

\section{REFERENCES}

1. Bearman G, Levenson R. Biological imaging spectroscopy. In: Vo-Dinh T, ed. Biomedical Photonics Handbook. 1st ed. Boca Raton, FL: CRC Press; 2003:8-1-8-5.

2. Hiraoka Y, Shimi T, Haraguchi T. Multispectral imaging fluorescence microscopy for living cells. Cell Struct Funct. 2002;27:367-374.

3. Levenson RM, Mansfield JR. Multispectral imaging in biology and medicine: slices of life. Cytometry A. 2006;69:748-758.

4. Mansfield JR, Hoyt C, Levenson RM. Visualization of microscopy-based spectral imaging data from multi-label tissue sections. Curr Protoc Mol Biol. 2008;84:14.19.1-14.19.15.

5. Zuba-Surma EK, Kucia M, Abdel-Latif A, Lillard JW Jr, Ratajczak MZ. The ImageStream System: a key step to a new era in imaging. Folia Histochem Cytobiol. 2007;45:279-290.

6. Mansfield JR, Gossage KW, Hoyt CC, Levenson RM. Autofluorescence removal, multiplexing, and automated analysis methods for in-vivo fluorescence imaging. J Biomed Opt. 2005;10:41207.

7. van der Loos CM. Multiple immunoenzyme staining: methods and visualizations for the observation with spectral imaging. J Histochem Cytochem. 2008;56:313-328.
8. Levenson RM, Lynch DT, Kobayashi H, Backer JM, Backer MV. Multiplexing with multispectral imaging: from mice to microscopy. ILAR J. 2008;49:78-88.

9. Hoffman RM. Imaging tumor angiogenesis with fluorescent proteins. APMIS. 2004;112:441-449.

10. Hoffman RM. Dual-color imaging of tumor angiogenesis. Methods Mol Biol. 2009;515:45-61.

11. Virostko J, Xie J, Hallahan DE, Arteaga CL, Gore JC, Manning HC. A molecular imaging paradigm to rapidly profile response to angiogenesis-directed therapy in small animals. Mol Imaging Biol. 2009;11:204-212.

12. Mayes P, Dicker D, Liu Y, El-Deiry W. Noninvasive vascular imaging in fluorescent tumors using multispectral unmixing. Biotechniques. Oct 2008; 45:459-460, 461-464.

13. Manning HC, Shay SD, Mericle RA. Multispectral molecular imaging of capillary endothelium to facilitate preoperative endovascular brain mapping. J Neurosurg. 2009;110:975-980.

14. Makhlouf H, Gmitro AF, Tanbakuchi AA, Udovich JA, Rouse AR. Multispectral confocal microendoscope for in vivo and in situ imaging. J Biomed Opt. 2008;13:044016.

15. Su J, Zhang J, Liu L, Huang Y, Mason RP. Exploring feasibility of multicolored $\mathrm{CdTe}$ quantum dots for in vitro and in vivo fluorescent imaging. $J$ Nanosci Nanotechnol. 2008;8:1174-1177.

16. Gao X, Cui Y, Levenson RM, Chung LW, Nie S. In vivo cancer targeting and imaging with semiconductor quantum dots. Nat Biotechnol. 2004;22:969-976.

17. Xing Y, Rao J. Quantum dot bioconjugates for in vitro diagnostics \& in vivo imaging. Cancer Biomark. 2008;4:307-319.

18. Tholouli E, Sweeney E, Barrow E, Clay V, Hoyland JA, Byers RJ. Quantum dots light up pathology. J Pathol. 2008;216:275-285.

19. Tholouli E, Hoyland JA, Di Vizio D, et al. Imaging of multiple mRNA targets using quantum dot based in situ hybridization and spectral deconvolution in clinical biopsies. Biochem Biophys Res Commun. 2006;348:628-636.

20. Fountaine TJ, Wincovitch SM, Geho DH, Garfield SH, Pittaluga S. Multispectral imaging of clinically relevant cellular targets in tonsil and lymphoid tissue using semiconductor quantum dots. Mod Pathol. 2006;19:1181-1191.

21. De Giorgi V, Massi D, Sestini S, Cicchi R, Pavone FS, Lotti T. Combined nonlinear laser imaging (two-photon excitation fluorescence microscopy, fluorescence lifetime imaging microscopy, multispectral multiphoton microscopy) in cutaneous tumours: first experiences. J Eur Acad Dermatol Venereol. 2009;23:314-316.

22. McGrath KE, Bushnell TP, Palis J. Multispectral imaging of hematopoietic cells: where flow meets morphology. J Immunol Methods. 2008;336:91-97.

23. George TC, Fanning SL, Fitzgerald-Bocarsly P, et al. Quantitative measurement of nuclear translocation events using similarity analysis of multispectral cellular images obtained in flow. J Immunol Methods. 2006;311:117-129.

24. George TC, Morrissey PJ, Cui C, Singh S, Bocarsly PF. Measurement of cytoplasmic to nuclear translocation. Curr Protoc Cytom. 2009 Jan;Chapter 9:Unit 9.28.

25. George TC, Basiji DA, Hall BE, et al. Distinguishing modes of cell death using the ImageStream multispectral imaging flow cytometer. Cytometry A. 2004; 59:237-245.

26. Henery S, George T, Hall B, Basiji D, Ortyn W, Morrissey P. Quantitative image based apoptotic index measurement using multispectral imaging flow cytometry: a comparison with standard photometric methods. Apoptosis. 2008;13:1054-1063.

27. Beum PV, Lindorfer MA, Hall BE, et al. Quantitative analysis of protein colocalization on B cells opsonized with rituximab and complement using the ImageStream multispectral imaging flow cytometer. J Immunol Methods. 2006;317:90-99.

28. Roblyer D, Richards-Kortum R, Sokolov K, et al. Multispectral optical imaging device for in vivo detection of oral neoplasia. J Biomed Opt. 2008;13:024019.

29. Hammer M, Konigsdorffer E, Liebermann C, et al. Ocular fundus autofluorescence observations at different wavelengths in patients with age-related macular degeneration and diabetic retinopathy. Graefes Arch Clin Exp Ophthalmol. 2008;246:105-114.

30. Pantalone D, Andreoli F, Fusi F, et al. Multispectral imaging autofluorescence microscopy in colonic and gastric cancer metastatic lymph nodes. Clin Gastroenterol Hepatol. 2007;5:230-236.

31. Jaganath R, Angeletti C, Levenson R, Rimm DL. Diagnostic classification of urothelial cells in urine cytology specimens using exclusively spectral information. Cancer. 2004;102:186-191.

32. Angeletti C, Harvey NR, Khomitch V, Fischer AH, Levenson RM, Rimm DL. Detection of malignancy in cytology specimens using spectral-spatial analysis. Lab Invest. 2005;85:1555-1564.

33. Mansoor I, Zalles C, Zahid F, Gossage K, Levenson RM, Rimm DL. Fine-needle aspiration of follicular adenoma versus parathyroid adenoma: the utility of multispectral imaging in differentiating lesions with subtle cytomorphologic differences. Cancer. 2008;114:22-26. 